\title{
The Relationship between Dialysis Metrics and Patient-Reported Cognition, Fatigue, and Physical Function
}

\author{
Daniel A. Sturgilla ${ }^{\text {Naveet Bal }}{ }^{a}$ Sneha Nagavally ${ }^{b}$ Dawn F. Wolfgram ${ }^{a}$ \\ aDivision of Nephrology, Department of Medicine, Medical College of Wisconsin, Milwaukee, WI, USA; \\ ${ }^{b}$ Center for Advancing Population Science, Medical College of Wisconsin, Milwaukee, WI, USA
}

\section{Keywords}

Dialysis · Patient-reported outcome · Function · Fatigue ·

PROMIS

\begin{abstract}
Introduction: The impact of achieving hemodialysis laboratory and hemodynamic quality metrics on patient-reported outcomes (PROs) is unknown. Objective: To determine if meeting dialysis laboratory quality of care measures is associated with improved PROs. Methods: In this cross-sectional study, we measured the relationship between dialysis patients' Patient Reported Outcome Measurement Information System (PROMIS) scores and commonly used dialysis quality of care measures. Results: PROMIS surveys were administered to 92 dialysis patients. The mean \pm SD scores demonstrated higher fatigue $(55.0 \pm 9.8)$ and lower physical function (37.9 \pm 7.9$)$ but similar cognition $(50.3 \pm 10.9)$ compared to general population normative scores of $50 \pm 10$. Dialysis patients meeting $\mathrm{Kt} / \mathrm{V}$ goals had no better scores than those who did not. Meeting the hemoglobin $(\mathrm{Hgb})$ value of $\geq 10 \mathrm{~g} / \mathrm{dL}$ was associated with a lower fatigue score, but no difference in cognitive or physical function scores. Meeting the serum albumin goal of $\geq 4.0 \mathrm{mg} / \mathrm{dL}$ was associated
\end{abstract}

with a higher physical function score but made no difference for cognitive function or fatigue score. As a continuous variable, a higher $\mathrm{Hgb}$ was associated with lower reported fatigue (HR $-1.7495 \%, \mathrm{Cl}[-3.09,-0.39])$, but no other measures were associated with PRO scores when adjusted for demographics and comorbidities. Conclusions: We found little association between measures currently used to assess the quality of dialysis care and PROs. Encouraging improved utilization of PROs and incorporating PROs into quality measurements might give a more robust assessment of quality of care. Future studies should assess the benefits of this approach.

(c) 2020 The Author(s)

Published by S. Karger AG, Basel

\section{Introduction}

Patients with end-stage renal disease (ESRD) suffer from a high burden of symptoms which are commonly associated with lower quality of life. The lower quality of life is further compounded by increased mortality and risk of hospitalizations $[1,2]$. The patient-reported symptoms that contribute to lower quality of life range from fatigue, muscle soreness, and cramps to difficulty concen- 
trating [3]. Monitoring and responding to patient-reported symptoms such as fatigue is noted to improve survival in oncology patients and may also benefit other complex patient populations such as dialysis patients [4]. The diversity of symptoms and the limited use of symptom measurement tools in the dialysis population have made measurements of dialysis patient symptoms difficult [5]. Fortunately, a broad range of domains including cognitive and physical function and symptoms such as fatigue can be measured with clinically validated tools known as patient-reported outcome (PRO) measures. PRO measures provide a standardized measure of symptoms and function that clinicians can easily review [6]. Understanding the relationship between PRO scores and clinical measures may identify if current measures of high-quality dialysis will improve patients' function and symptoms.

Currently, the assessment of dialysis quality focuses largely on laboratory data rather than patient symptoms. The large dialysis organizations in the USA focus on several laboratory values as a measure of quality of dialysis care such as dialysis adequacy (urea clearance and $\mathrm{Kt} / \mathrm{V}$ ), hemoglobin (Hgb), and serum albumin (albumin), factors known to associate with mortality [7-9]. In fact, until very recently, the dialysis adequacy measure $(\mathrm{Kt} / \mathrm{V})$ was included in the Centers for Medicare and Medicaid Services (CMS) quality incentive program that determines a facility's payments for providing dialysis, with not achieving the Kt/V target leading to lower payments to facilities for all the dialysis services they provided. However, it is not known whether achieving these metrics is associated with better health-related quality of life. The Kidney Disease Quality of Life 36 (KDQOL-36), a PRO measure, is given annually to all ESRD patients, and it is time consuming, taking up to $30 \mathrm{~min}$ to complete; it has low completion rates (36\%) in clinical practice [10]. A questionnaire that is completed quickly and thus can increase participation rates, but still provides needed information will help determine if the above metrics are associated with better health-related quality of life.

The National Institutes of Health (NIH) Patient Reported Outcome Measurement Information System (PROMIS) was created to better assess PROs in chronic disease [11]. PROMIS surveys can be administered in $<3$ min via computer adaptive testing (CAT), a format that uses item response theory. These surveys have high reliability and validity, good discrimination within levels of impairment, and the precision needed to track an individual's change over time [12]. Recent reports indicate that PROMIS measures have better reliability than the Medical Outcomes Study 12 item Short Form Health Sur- vey which make up questions 1-12 in the KDQOL-36 [6]. In the kidney transplant population, a comparison between a 4-question per domain PROMIS survey had strong correlation with KDQOL-36 with improved ease of use and lower responder burden [13]. To date, no studies have used PROMIS surveys to explore whether meeting laboratory-based metrics such as dialysis adequacy, albumin, and $\mathrm{Hgb}$ is associated with improved PROMIS scores in adults undergoing dialysis. We chose the laboratory values as they are associated with mortality and not meeting the designated targets can lead to significant changes in the dialysis prescription and medications. The PROs we focused on are known to be commonly impaired in dialysis patients [14]. In this cross-sectional study of patients with ESRD on dialysis, we measured the relationship between frequently monitored and required laboratory targets and symptoms associated with cognitive function, fatigue, and physical function.

\section{Materials and Methods}

\section{Dialysis Survey Administration}

As part of clinical care, patients receiving in-center/conventional hemodialysis (HD), home hemodialysis (HHD), or peritoneal dialysis (PD) completed 3 NIH PROMIS surveys via CAT on an iPad. Survey administration was done during HD treatments for those patients on in-center HD and to home dialysis patients (PD and HHD) during the monthly clinic visit. The survey administrator asked the patient the survey questions and recorded the patient's answers. This maintained survey quality as it ensured that the question was read correctly (from the iPad screen) for patients who may have had difficulty reading or were visually impaired and the correct answer was clicked as this can be difficult for some HD patients due to hand weakness or constraints in movement due to the use of the vascular access. For all patients we collected demographic data, cause of ESRD, comorbid conditions, and dialysis laboratory parameters of $\mathrm{Hgb}$, albumin, and Kt/V. The laboratories were all measured in the same month as the survey administration. This study was approved by the Medical College of Wisconsin Institutional Review Board.

\section{Statistical Analysis}

We performed descriptive statistics to summarize demographics and PROMIS score range in our cohort. We compared mean scores between groups meeting or not meeting the targets employed by the participating dialysis units. The Kt/V target was selected based on Centers for Medicare and Medicaid Services Quality Incentive Program (CMS QIP) metric of standard pool Kt/V $\geq 1.4$ for $\mathrm{HD}, \geq 1.7$ for $\mathrm{PD}$, and $\geq 2.0$ for HHD which determines CMS payment amounts. The targets of $\mathrm{Hgb} \geq 10 \mathrm{~g} / \mathrm{dL}$ and serum albumin $\geq 4.0 \mathrm{~g} / \mathrm{dL}$ are the current targets used in the dialysis units where the study was conducted. These targets are used by the large dialysis organizations to rank the quality of care in the different units. We used $t$ tests to compare mean scores between groups and also analysis of covariance to test the effects of the dialysis metrics 

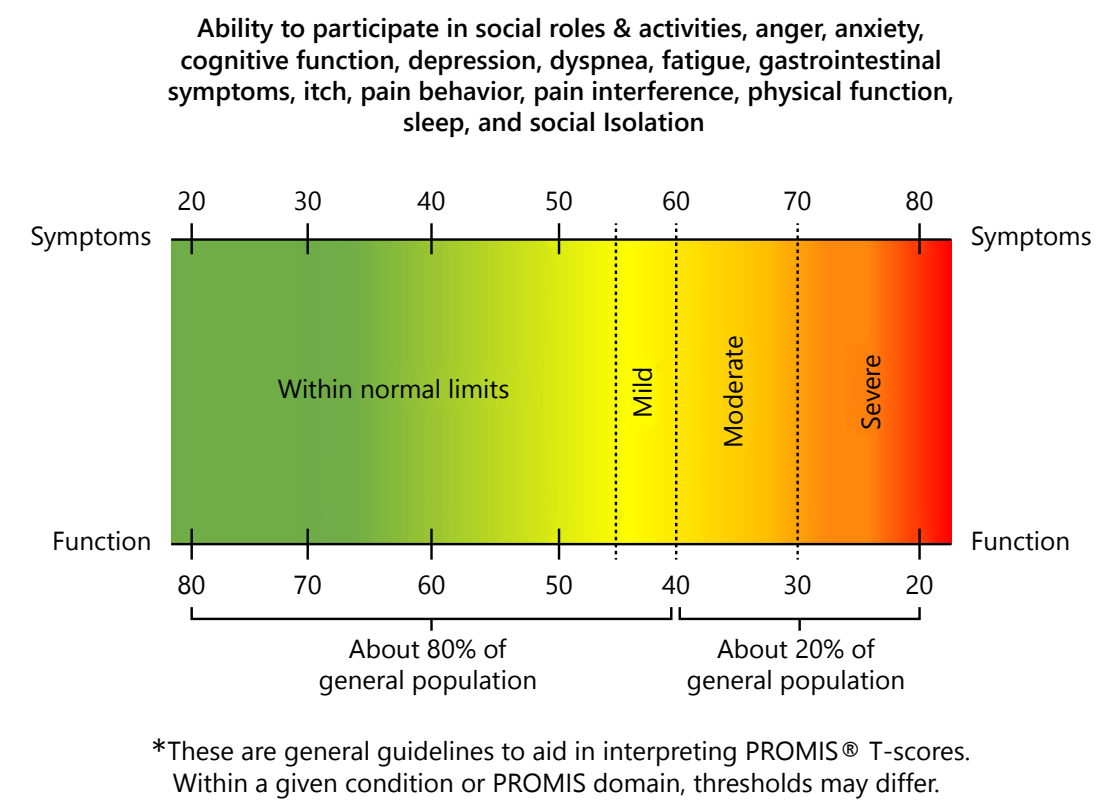

Fig. 1. A higher score means that the patient has reported more of that symptoms or function. A higher physical function score would be they report better physical function and a higher fatigue score indicates they report more fatigue. All PROMIS scores have a mean of 50 and standard deviation of 10 in the general population.

and age on the PROMIS scores. We included age due to the known effect on the domains being measured. Due to the small cohort size, we also used a linear regression model followed by an adjusted linear regression to evaluate the continuous relationship between fatigue, cognition, physical function PROMIS scores, and $\mathrm{Kt} / \mathrm{V}, \mathrm{Hgb}$, and albumin, which would have greater ability to detect to any relationship between the laboratories and PROMIS scores. Age, gender, race, and comorbidities (hypertension and diabetes) were included in the adjusted model as covariates. RStudio statistical software was used for analysis.

\section{PROMIS Score Interpretation}

The PROMIS CAT survey uses item response theory and has at minimum 4 questions with sequential questions changing based on previous answers up to the maximum 12 questions. T-scores are automatically calculated for the CAT form and with general population reference of 50 with standard deviation of 10 [15]. A higher T-score indicates more of the concept being measured (Fig. 1). For example, a person with a score of 60 would have fatigue 1 standard deviation worse than the average person.

\section{Results}

We obtained survey data from 92 dialysis patients. The cohort was $40.2 \%$ female, $42.9 \%$ White, and $46.2 \%$ African American, and the mean age was $56.1 \pm 14.8$ years. Diabetes and hypertension were the most common causes of ESRD at 40.2 and $19.5 \%$, respectively. The majority $(77.2 \%)$ of dialysis patients received in-center/conventional $\mathrm{HD}, 7.6 \%$ received $\mathrm{HHD}$, and $15.2 \%$ received $\mathrm{PD}$. The most prevalent comorbidities were hypertension and diabetes, followed by congestive heart failure and coronary artery disease. See Table 1 for characteristics of the cohort.

When comparing in-center $\mathrm{HD}$ to home dialysis (PD and HHD), we found no significant difference in fatigue (55.6 vs. 53.7, $p=0.38$ ) or cognition ( 49.57 vs. $52.07, p=$ $0.36)$. The home dialysis group reported better physical function than the in-center HD group (41.2 vs. 36.8, $p=$ $0.03)$.

The mean PROMIS scores were 50.3 \pm 10.9, $55.0 \pm 9.8$, and $37.9 \pm 7.9$ for cognition, fatigue, and physical function, respectively. These scores indicate that the dialysis cohort reported higher fatigue and lower levels of physical function compared to the general population. The mean $\mathrm{Hgb}$ was $11.0 \pm 1.5 \mathrm{~g} / \mathrm{dL}$, albumin was $3.8 \pm 0.5 \mathrm{~g} /$ $\mathrm{dL}$, and $\mathrm{Kt} / \mathrm{V}$ was $1.7 \pm 0.4$. The proportion of patients meeting each laboratory metric target is noted in Table 1.

There were no significant differences in PROMIS scores for any domain based on the patient achieving the goal Kt/V (target for dialysis adequacy). Patients whose $\mathrm{Hgb}$ was at least $10 \mathrm{~g} / \mathrm{dL}$ had lower mean fatigue scores 
Table 1. Demographic and baseline data for dialysis cohort $(n=$ 92)

\begin{tabular}{|c|c|}
\hline Variable & Values \\
\hline Age, mean $\pm S D$ & $56.1 \pm 14.8$ \\
\hline Female gender, $n(\%)$ & $37(40.2)$ \\
\hline \multicolumn{2}{|l|}{ Race, $n(\%)$} \\
\hline White & $39(42.9)$ \\
\hline African American & $42(46.1)$ \\
\hline Others & $10(10.9)$ \\
\hline \multicolumn{2}{|l|}{ Cause of ESRD, $n(\%)$} \\
\hline Diabetes mellitus & $37(40.2)$ \\
\hline Glomerulonephritis & $14(15.2)$ \\
\hline Hypertension & $18(19.6)$ \\
\hline Others & $13(14.1)$ \\
\hline \multicolumn{2}{|l|}{ Comorbidities, $n(\%)$} \\
\hline Hypertension & $84(91.3)$ \\
\hline Diabetes & $49(53.3)$ \\
\hline Stroke & $10(10.9)$ \\
\hline Coronary artery disease & $15(16.3)$ \\
\hline Congestive heart failure & $28(30.4)$ \\
\hline Liver cirrhosis & $4(4.4)$ \\
\hline Peripheral vascular disease & $8(8.7)$ \\
\hline Depression & $10(10.9)$ \\
\hline Dementia or mild cognitive impairment & $7(7.6)$ \\
\hline \multicolumn{2}{|l|}{ Dialysis modality, $n(\%)$} \\
\hline In-center/conventional hemodialysis & $71(77.2)$ \\
\hline Home hemodialysis & $7(7.6)$ \\
\hline Peritoneal dialysis & $14(15.2)$ \\
\hline \multicolumn{2}{|l|}{ Laboratory (mean \pm SD) } \\
\hline Hemoglobin, g/dL & $11.0 \pm 1.5$ \\
\hline Albumin, g/dL & $3.8 \pm 0.5$ \\
\hline $\mathrm{Kt} / \mathrm{V}$ & $1.7 \pm 0.4$ \\
\hline \multicolumn{2}{|l|}{ Proportion meeting laboratory target, $n(\%)$} \\
\hline Hemoglobin $\geq 10 \mathrm{~g} / \mathrm{dL}$ & $72(78.2)$ \\
\hline Albumin $\geq 4.0 \mathrm{~g} / \mathrm{dL}$ & $36(39.1)$ \\
\hline $\mathrm{Kt} / \mathrm{V}$ goal & $68(73.9)$ \\
\hline
\end{tabular}

ESRD, end-stage renal disease; g/dL, grams per deciliter.

than those whose $\mathrm{Hgb}$ was lower than $10 \mathrm{~g} / \mathrm{dL}$ (53.4 vs. $61.1, p<0.01$ ), but cognitive and physical function did not differ. There was no difference in fatigue or cognitive function scores for those meeting albumin targets compared to those who did not, but physical function scores were higher $(40.8$ vs. $36.1, p<0.01)$ (see Table 2$)$.

For the total dialysis cohort (see Table 3 ), unadjusted regression showed significant associations between higher $\mathrm{Hgb}$ and lower reported fatigue score with a change of $-1.86(95 \%$ CI $[-3.16,-0.55])$ points for each increase of $1 \mathrm{~g} / \mathrm{dL}$ of $\mathrm{Hgb}$. This relationship remained significant in the adjusted model with a change of -1.74 points $/ 1 \mathrm{~g} / \mathrm{dL}$ (95\% CI $[-3.09,-0.39])$. Higher albumin was associated with greater reported physical function with a $4.2(95 \%$
CI $[0.62,7.62])$ point increase in score for every $1 \mathrm{~g} / \mathrm{dL}$ increase in albumin, although this association was not significant in the adjusted model.

\section{Discussion/Conclusion}

In our dialysis cohort, traditional dialysis targets that are used to measure quality of care were seldom associated with PROs. Our study found no improvement in PROs of cognition, fatigue, or physical function based on meeting Kt/V goals and only found that meeting the $\mathrm{Hgb}$ target of $10 \mathrm{~g} / \mathrm{dL}$ and serum album of $4.0 \mathrm{~g} / \mathrm{dL}$ was associated with fatigue and physical function, respectively. It is important to determine if laboratory targets may be associated with PROs since our cohort demonstrated much higher fatigue levels and decreases in physical function compared to general population scores. Interestingly, the cohort did not report decreased cognition, and there was no difference in reported cognition for those meeting the target Kt/V versus those who did not. This is a noteworthy finding given the vast literature indicating the high degree of cognitive impairment as measured by objective testing in the dialysis population and the generally accepted theory that poor clearance of uremic solutes (measured by $\mathrm{Kt} / \mathrm{V}$ ) can contribute to cognitive dysfunction [16]. Overall, our study used contemporary dialysis quality targets in a representative sample of US dialysis patients and demonstrated a lack of association with PROs.

By evaluating cutoff values consistent with the CMS and dialysis organization set established goals of albumin $\geq 4.0 \mathrm{~g} / \mathrm{dL}, \mathrm{Hgb} \geq 10 \mathrm{~g} / \mathrm{dL}$, and $\mathrm{Kt} / \mathrm{V}$, we were able to determine which laboratory metrics are also associated with improved PROs. The differences in scores based on meeting $\mathrm{Hgb}$ and albumin targets are consistent with meaningful differences in PROMIS scores based on prior literature. Meeting the Hgb target was associated with an 8 -point difference in fatigue scores, nearly 1 standard deviation, and difference in score that often motivates patients to accept a change in treatment [17]. Even the difference in physical function score of 4 points is within the minimally important difference of 3-5 points noted for that scale [18]. The importance of these 2 targets is not surprising based on prior literature. The association of anemia and self-report fatigue levels is consistent with fatigue levels assessed by using the KDQOL, although this study did not evaluate the target of $10 \mathrm{~g} / \mathrm{dL}$ [19]. Lower albumin levels are associated with lower self-report physical activity as measured by the maximum activity score; however, this study only looked at albumin as a continu- 
Table 2. Comparing mean PROMIS scores between those that met dialysis laboratory targets and those who did not

\begin{tabular}{|c|c|c|c|c|c|c|c|}
\hline \multirow[t]{2}{*}{ Metric } & \multirow[t]{2}{*}{$N$} & \multicolumn{2}{|c|}{ Cognitive T-score } & \multicolumn{2}{|c|}{ Fatigue T-scores } & \multicolumn{2}{|c|}{ Physical function T-scores } \\
\hline & & mean $(\mathrm{SD})$ & $p$ value & mean $(\mathrm{SD})$ & $p$ value & mean $(\mathrm{SD})$ & $p$ value \\
\hline \multicolumn{8}{|l|}{$\mathrm{Kt} / \mathrm{V}^{\mathrm{a}}$} \\
\hline At goal & 68 & $50.9(11.4)$ & \multirow[t]{2}{*}{0.26} & $54.7(9.80)$ & \multirow[t]{2}{*}{0.88} & $38.3(8.30)$ & \multirow[t]{2}{*}{0.56} \\
\hline Below goal & 23 & $48.2(9.04)$ & & $55.1(9.39)$ & & $37.2(6.85)$ & \\
\hline \multicolumn{8}{|l|}{ Albumin } \\
\hline$\geq 4.0 \mathrm{~g} / \mathrm{dL}$ & 36 & $50.8(11.0)$ & \multirow[t]{2}{*}{0.72} & $53.7(9.37)$ & \multirow[t]{2}{*}{0.30} & $40.8(6.96)$ & \multirow[t]{2}{*}{$<0.01$} \\
\hline$<4.0 \mathrm{~g} / \mathrm{dL}$ & 56 & $50.0(10.9)$ & & $55.9(10.1)$ & & $36.1(8.04)$ & \\
\hline \multicolumn{8}{|l|}{ Hemoglobin } \\
\hline$\geq 10.00 \mathrm{~g} / \mathrm{dL}$ & 72 & $50.5(11.1)$ & \multirow[t]{2}{*}{0.73} & $53.4(9.72)$ & \multirow[t]{2}{*}{$<0.01$} & $38.7(7.95)$ & \multirow[t]{2}{*}{0.08} \\
\hline$<10.00 \mathrm{~g} / \mathrm{dL}$ & 20 & $49.6(10.2)$ & & $61.1(7.58)$ & & $35.2(7.47)$ & \\
\hline
\end{tabular}

Significant differences in PROMIS scores between those that met the target versus those that did not are shown in bold. PROMIS, Patient Reported Outcome Measurement Information System; HD, hemodialysis; HHD, home hemodialysis; PD, peritoneal dialysis. a The Kt/V goal for in-center HD was 1.4 (standard pool), for PD was 1.7 (weekly), and for HHD was 2.0 (weekly). There was 1 subject who did not have an accurate Kt/V during the month that the PROMIS scores were measured.

Table 3. Change in PROMIS score based on laboratory metric

\begin{tabular}{|c|c|c|c|c|c|c|}
\hline & \multicolumn{2}{|l|}{ Fatigue } & \multicolumn{2}{|c|}{ Physical functioning } & \multicolumn{2}{|c|}{ Cognitive functioning } \\
\hline Albumin & $-2.48(-6.91,1.94)$ & $-2.74(-7.30,1.82)$ & $4.12(0.62,7.62)$ & $3.51(-0.12,7.14)$ & $1.12(-3.82,6.05)$ & $0.85(-4.24,5.94)$ \\
\hline $\mathrm{Kt} / \mathrm{V}$ & $-0.23(-0.77,0.31)$ & $-0.36(-0.93,0.20)$ & $0.43(-0.01,0.86)$ & $0.39(-0.07,0.84)$ & $0.49(-0.11,1.10)$ & $0.54(-0.10,1.17)$ \\
\hline
\end{tabular}

Change in PROMIS score is per $1 \mathrm{~g} / \mathrm{dL}$ of $\mathrm{Hgb}, 1 \mathrm{~g} / \mathrm{dL}$ of albumin, and 0.1 unit Kt/V. Bold text indicates significance at $p<0.05$ level. PROMIS, Patient Reported Outcome Measurement Information System. ${ }^{\text {a }}$ Adjusted for age, gender, race, and comorbidities (hypertension and diabetes mellitus).

ous variable and did not examine meeting the albumin target of $4.0 \mathrm{~g} / \mathrm{dL}[20]$. In contrast to prior literature, our study provides new information on whether meeting current goals is associated with improved scores rather than just noting that higher $\mathrm{Hgb}$ and albumin in general are associated with lower fatigue and improved physical function.

Surprisingly, meeting Kt/V targets was not associated with any PROs nor was there any association when $\mathrm{Kt} / \mathrm{V}$ was evaluated as a continuous variable. This brings into question the use of a Kt/V target to measure dialysis adequacy, which should center on the patient's overall wellbeing [21]. Trying to attain the Kt/V target may lead to prolonging time on dialysis or increasing fluid removal, which will both result in higher Kt/V. However, these changes can result in more dialysis-associated symptoms such as fatigue and cramping. The focus on meeting the $\mathrm{Kt} / \mathrm{V}$ target may lead to significant changes for those on home dialysis since not achieving $\mathrm{Kt} / \mathrm{V}$ goals is a common reason cited for changing from home dialysis to in-center HD [22]. This may be important as the home modality was associated with improved report of physical function in our cohort. The lack of improvement in PROs for those meeting Kt/V goals may indicate that the goals used for $\mathrm{Kt} / \mathrm{V}$ may need re-evaluation or that PROs should be used in conjunction with $\mathrm{Kt} / \mathrm{V}$ to give an overall picture of dialysis quality. The current Kt/V targets are based on trials done decades ago on very different dialysis populations compared to current dialysis patients, and only accounted for the outcome of mortality [23, 24].

Our finding of lower self-reported physical function and greater fatigue complements the current literature; however, the reports of preserved cognitive function provide new insight in how patients view their cognitive function. In dialysis patients, objective measures of physical function such as increased sarcopenia and decreased gait speed are consistent with the lower reported physical function ability $[25,26]$. Despite evidence that patients 
receiving dialysis suffer from cognitive impairment on formal cognitive testing, our cohort self-reported intact cognitive function $[27,28]$. It is possible that participants were more hesitant to admit to cognitive issues as they may feel more stigma associated with cognitive impairment compared to impairment of physical function or increased fatigue. Both denial and unawareness of cognitive impairment are possible reasons for the self-reported intact cognition in our cohort as they are known limitations in self-report of cognitive function [29].

This cross-sectional study has limitations. The primary limitation is that the study is small and single center. However, our population is representative of the current US dialysis population. This limits our ability to find some more minor associations due to lack of power. However, we were able to see meaningful differences in PRO scores for both Hgb and albumin. Second, we have no information on the relationship between the patient's subjective report and objectively measured cognitive or physical function. This prevents us from determining if there are more objective changes in physical function or cognition based on meeting laboratory metrics. Finally, we have no longitudinal data to report, so we are unable to determine if a change from meeting the goal to not meeting the goal or vice versa are associated with changes in the PRO scores.

Using PROMIS measurements of fatigue, physician function, and cognition in a dialysis cohort, we found that achieving targets for $\mathrm{Hgb}$ and albumin, which are associated with mortality, also are associated with improved PROs in dialysis patients. This may provide additional information and incentive to patients on the relevance of the monitored laboratory parameters. However, achievement of the set goals for some commonly used metrics, such as $\mathrm{Kt} / \mathrm{V}$, may not be relevant in PROs and in certain patient groups may need to be less emphasized in order to provide patient-centered care. The use of PROMIS surveys can provide valuable patient-centered care that is not included in current laboratory measures that evaluate dialysis quality. We would encourage future studies to incorporate PROMIS survey scores as a clinically and patient-relevant outcome in addition to laboratory markers when evaluating interventions aimed at improving the quality of dialysis care.

\section{Acknowledgements}

The authors thank Dr. Jeffrey Whittle for his help in organization and editing of the paper and Dr. Rebekah Walker for her statistical input.

\section{Statement of Ethics}

The study protocol has been approved by the Medical College of Wisconsin committee on human research (IRB approval number: PRO 00030048).

\section{Conflict of Interest Statement}

The authors have no conflicts of interest to declare.

\section{Funding Sources}

Dawn Wolfgram is supported by the National Institute of Diabetes, Digestive and Kidney Diseases of the National Institutes of Health under Award Number K23DK113119.

\section{References}

1 Hall RK, Luciano A, Pieper C, Colon-Emeric CS. Association of Kidney Disease Quality of Life (KDQOL-36) with mortality and hospitalization in older adults receiving hemodialysis. BMC Nephrol. 2018;19(1):11.

2 Mapes DL, Lopes AA, Satayathum S, McCullough KP, Goodkin DA, Locatelli F, et al. Health-related quality of life as a predictor of mortality and hospitalization: the Dialysis Outcomes and Practice Patterns Study (DOPPS). Kidney Int. 2003;64(1):339-49.

3 Abdel-Kader K, Unruh ML, Weisbord SD. Symptom burden, depression, and quality of life in chronic and end-stage kidney disease. Clin J Am Soc Nephrol. 2009;4(6):1057-64.
4 Basch E, Deal AM, Dueck AC, Scher HI, Kris MG, Hudis C, et al. Overall survival results of a trial assessing patient-reported outcomes for symptom monitoring during routine cancer treatment. JAMA. 2017;318(2):197-8

5 Flythe JE, Powell JD, Poulton CJ, Westreich KD, Handler L, Reeve BB, et al. Patient-reported outcome instruments for physical symptoms among patients receiving maintenance dialysis: a systematic review. Am J Kidney Dis. 2015;66(6):1033-46.

6 Peipert JD, Hays RD. Using patient-reported measures in dialysis clinics. Clin J Am Soc Nephrol. 2017;12(11):1889-91.
7 Owen WF Jr, Lew NL, Liu Y, Lowrie EG, Lazarus JM. The urea reduction ratio and serum albumin concentration as predictors of mortality in patients undergoing hemodialysis. N Engl J Med. 1993;329(14):1001-6.

8 Toft G, Heide-Jorgensen U, van Haalen H, James G, Hedman K, Birn H, et al. Anemia and clinical outcomes in patients with non-dialysis dependent or dialysis dependent severe chronic kidney disease: a Danish populationbased study. J Nephrol. 2020;33(1):147-56.

9 Thorp ML, Johnson ES, Yang X, Petrik AF, Platt R, Smith DH. Effect of anaemia on mortality, cardiovascular hospitalizations and end-stage renal disease among patients with chronic kidney disease. Nephrology. 2009;14(2):240-6. 
10 Marrufo G, Negrusa B, Ullman D, Melin C, Hirth R, Messana J, et al. Comprehensive End-Stage Renal Disease Care (CEC) Model. Performance Year 1 Annual Evaluation Report. Falls Church: Lewin Group; 2017.

11 Cook KF, Jensen SE, Schalet BD, Beaumont JL, Amtmann D, Czajkowski S, et al. PROMIS measures of pain, fatigue, negative affect, physical function, and social function demonstrated clinical validity across a range of chronic conditions. J Clin Epidemiol. 2016; 73:89-102.

12 Fries JF, Bruce B, Cella D. The promise of PROMIS: using item response theory to improve assessment of patient-reported outcomes. Clin Exp Rheumatol. 2005;23(5 Suppl 39):S53-7.

13 Tang E, Ekundayo O, Peipert JD, Edwards N, Bansal A, Richardson C, et al. Validation of the Patient-Reported Outcomes Measurement Information System (PROMIS)-57 and -29 item short forms among kidney transplant recipients. Qual Life Res. 2019;28(3): 815-27.

14 Carmichael P, Popoola J, John I, Stevens PE, Carmichael AR. Assessment of quality of life in a single centre dialysis population using the KDQOL-SF questionnaire. Qual Life Res. 2000;9(2):195-205.

15 Cella D, Riley W, Stone A, Rothrock N, Reeve B, Yount S, et al. The Patient-Reported Outcomes Measurement Information System (PROMIS) developed and tested its first wave of adult self-reported health outcome item banks: 2005-2008. J Clin Epidemiol. 2010; 63(11):1179-94.
16 Drew DA, Weiner DE, Sarnak MJ. Cognitive impairment in CKD: pathophysiology, management, and prevention. Am J Kidney Dis. 2019;74(6):782-90.

17 Kozlowski AJ, Cella D, Nitsch KP, Heinemann AW. Evaluating individual change with the Quality of Life in Neurological Disorders (Neuro-QoL) Short Forms. Arch Phys Med Rehabil. 2016;97(4):650-4 e8.

18 Yost KJ, Eton DT, Garcia SF, Cella D. Minimally important differences were estimated for six Patient-Reported Outcomes Measurement Information System-Cancer scales in advanced-stage cancer patients. J Clin Epidemiol. 2011;64(5):507-16.

19 Finkelstein FO, Story K, Firanek C, Mendelssohn D, Barre P, Takano T, et al. Health-related quality of life and hemoglobin levels in chronic kidney disease patients. Clin J Am Soc Nephrol. 2009;4(1):33-8.

20 Anand S, Chertow GM, Johansen KL, Grimes B, Kurella Tamura M, Dalrymple LS, et al. Association of self-reported physical activity with laboratory markers of nutrition and inflammation: the comprehensive dialysis study. J Ren Nutr. 2011;21(6):429-37.

21 Perl J, Dember LM, Bargman JM, Browne T, Charytan DM, Flythe JE, et al. The use of a multidimensional measure of dialysis adequacy-moving beyond small solute kinetics. Clin J Am Soc Nephrol. 2017;12(5):839-47.
22 Boissinot L, Landru I, Cardineau E, Zagdoun E, Ryckelycnk JP, Lobbedez T. Is transition between peritoneal dialysis and hemodialysis really a gradual process? Perit Dial Int. 2013; 33(4):391-7.

23 Lowrie EG, Laird NM, Parker TF, Sargent JA Effect of the hemodialysis prescription of patient morbidity: report from the National Cooperative Dialysis Study. N Engl J Med. 1981; 305(20):1176-81.

24 Eknoyan G, Beck GJ, Cheung AK, Daugirdas JT, Greene T, Kusek JW, et al. Effect of dialysis dose and membrane flux in maintenance hemodialysis. N Engl J Med. 2002;347(25): 2010-9.

25 Kim JK, Choi SR, Choi MJ, Kim SG, Lee YK, Noh JW, et al. Prevalence of and factors associated with sarcopenia in elderly patients with end-stage renal disease. Clin Nutr. 2014; 33(1):64-8.

26 Johansen KL, Chertow GM, da Silva M, Carey S, Painter P. Determinants of physical performance in ambulatory patients on hemodialysis. Kidney Int. 2001;60(4):1586-91.

27 Murray AM, Tupper DE, Knopman DS, Gilbertson DT, Pederson SL, Li S, et al. Cognitive impairment in hemodialysis patients is common. Neurology. 2006;67(2):216-23.

28 Kurella M, Chertow GM, Luan J, Yaffe K. Cognitive impairment in chronic kidney disease. J Am Geriatr Soc. 2004;52(11):1863-9.

29 Weintraub S, Carrillo MC, Farias ST, Goldberg TE, Hendrix JA, Jaeger J, et al. Measuring cognition and function in the preclinical stage of Alzheimer's disease. Alzheimers Dement. 2018;4:64-75. 